\title{
Marketing Social para promover el liderazgo en la comunidad educativa de pre-grado en la Universidad Tecnológica Empresarial de Guayaquil
}

\section{Social Marketing to promote leadership in the pre-graduate educational community at the Universidad Tecnologica Empresarial de Guayaquil}

\author{
MSc, Diego Roberto Núñez Domínguez ${ }^{1}$ \\ dnulez@itsvr.edu.ec \\ PHD, Milton Rafael Maridueña Arroyave ${ }^{2}$ \\ mmaridueña@itsvr.edu.ec
}

Recibido: 1/12/2018; Aceptado: 1/02/2019

\begin{abstract}
RESUMEN
Desde el periodo colonial en 1603 , en el país se creó la Universidad San Fulgencio y las carreras que existían se basaban en el Derecho Canónico, Teología y Arte. El liderazgo de la época era generado por la jerarquía del reinado de turno desde España, así también por los militares y sacerdotes, conocido como líderes de cargo. En los siglos XVII (1600) y XVIII (1800) fueron gobernados por esta clase de autoridad, la cual tenía al pueblo atemorizado y oprimido. En el siglo XIX la educación superior en el Ecuador, tomó mucha importancia, siendo el Presidente Vicente Rocafuerte (1836), quien definió el nombre de la Universidad Central del Ecuador, como la primera universidad pública del país. En ella se impartían la Medicina, Ciencia, Arte, Derecho; sin embargo, desde entonces la Universidad tuvo inestables momentos, siendo clausurada por motivos políticos, por gobiernos como: García Moreno (1869) por seis años, Ignacio de Veintimilla (1880) por tres años, Velasco Ibarra (1934) reabierto el mismo año, Aurelio Mosquera Narváez (1939) reabierto el mismo año, los militares (1964), Velasco Ibarra (1970) la cierra por nueve años y en 1979 apertura las puertas bajo vigilancia de triunvirato. El liderazgo del siglo XIX sigue siendo por cargo y poder y no existía una libertad real y honesta para desarrollar al país con líderes que realmente se preocupen del pueblo, solo existían sus principios y su propio beneficio. Hoy, en el siglo XXI donde la palabra líder ha emprendido un significado mucho más importante en la vida de las personas, tanto en lo político, empresarial, sociedad, comunidad, así también en la innovación y negocios independientes, donde hablar de autoridad ya no es una palabra de poder o dictadura, se la entiende como una dirección crucial para la población, dirigir de forma correcta en paz y armonía a la sociedad.
\end{abstract}

1 Universidad Tecnológica Empresarial de Guayaquil. Ecuador

2 Universidad Tecnológica Empresarial de Guayaquil. Ecuador

Revista científica Ciencia y Tecnología Vol 19 No 22 págs. 12-17 http://cienciaytecnologia.uteg.edu.ec 
Palabras Clave: universidad, liderazgo, líder por cargo

\begin{abstract}
From the colonial in 1603 the University San Fulgencio was created in Ecuador. Careers were based on Canon Law, Theology and Arts. Leadership of the time was generated by the King of Spain, as well as by the military and priests. In the seventeenth (1600) and XVIII (1800) centuries territories were reigned by the King's authority and people were frightened and oppressed. In the nineteenth century higher education in Ecuador took a lot of importance for the political state. President Vicente Rocafuerte (1836), defined the name as Central University of Ecuador and as the first public university in the country. Careers such as Medicine Science, Art, Law were offered. However since then the University had unstable moments. It was closed by several governments such as; García Moreno (1869) for six years, Ignacio de Veintimilla (1880) for three years, Velasco Ibarra (1934) reopened the same year, Aurelio Mosquera Narvaez (1939) reopened the same year, the military (1964), Velasco Ibarra (1970) closed it for nine years, and in 1979 opened the doors under triumvirate surveillance. As discussed above, the leadership of the nineteenth century remains based in political power and there was no real and honest freedom to develop the nation with leaders who really care about the people; they only seek their own benefit. Today, in the $21^{\text {st }}$ century where the word leader has undertaken a much more important meaning in people's lives, both in politics, business, society, community, as well as in innovation and independent business, where to speak of authority and it is not a word of power or dictatorship symptom, it is currently understood that its direction should be crucial for the population, leading society in peace and harmony.
\end{abstract}

Keywords: university, leadership, leader by position

\title{
Introducción
}

Una de las bases más importante y que por derecho que debe tener el ser humano es la educación, donde la universidad es la madre nodriza de los estudiantes que adquieren este conocimiento en la carrera que hayan escogido por libre decisión. Sin embargo, existe un gran porcentaje de personas que tienen un título de tercer nivel pero carecen de liderazgo. Esto es un problema muy serio dentro de la sociedad, porque en la actualidad ser titulado de la profesión que se haya escogido ya no es una carta de presentación para ninguna empresa, organización, comunidad a la que se quiere pertenecer, ni para emprender un negocio sino sabes cómo liderar.

Muchas universidades, en sus publicidades dicen formar líderes del mañana; sin embargo, no lo es (El Universo, 2014). Forman en la parte teórica y práctica, que en muchas ocasiones la teoría es solo el porcentaje minoritario que queda como experiencia durante todo el transcurso de la carrera que escoge el alumno y la práctica obtiene una mayor participación de reconocimiento en lo profesional.

Existen varias características del liderazgo que no están siendo empleadas en los alumnos que se gradúan en las universidades de pre-grado como: responsabilidad al cambio positivo de las personas, mejoramiento de las relaciones interpersonales, incrementar el hábito de lectura, generar actitud positiva, comunicación eficaz, autodisciplina e influir positivamente en las personas. Estas son cualidades que hoy 


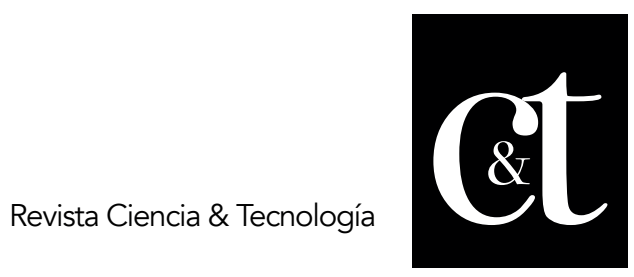

No. 22, 30 de abril de 2019

ISSN impreso: 1390 - 6321

ISSN online: 2661 - 6734

en día buscan los empleadores de empresas para ser parte de sus instituciones.

Existe una infinidad de alumnos con excelentes calificaciones que han terminado la universidad, pero no saben cómo desenvolverse en la sociedad. Esto les ocasiona un fracaso tanto personal como profesional, porque no encontraron ese liderazgo que necesitaban para una entrevista de trabajo, cerrar un negocio, dirigirse a un público determinado, capacitar, enseñar, etc.

Hay un sinnúmero de escenarios que sin el liderazgo que debe tener una persona, podría estar pensando que los estudios realizados no le enseñaron a preparase para el mundo competitivo, cuando el problema real es otro y no lo que aprendieron en su carrera universitaria.

Es importante que la universidad sea capaz en el nivel de pre-grado de identificar en cada alumno sus cualidades, y desarrollar el potencial del futuro profesional que están creando. Esto también generará fidelidad del alumnado e impactará en la sociedad.

\section{Desarrollo}

Marco Conceptual

Marketing: Para Kotler \& Armstrong (2003) "el marketing es un proceso social y administrativo mediante el cual grupos e individuos obtienen lo que necesitan y desean a través de generar, ofrecer e intercambiar productos de valor con sus semejantes".

Según McCarthy (1960), "el marketing es la realización de aquellas actividades que tienen por objeto cumplir las metas de una organización, al anticiparse a los requerimientos del consumidor o cliente y al encauzar un flujo de mercancías aptas a las necesidades y los servicios que el productor presta al consumidor o cliente".

Marketing Social: De acuerdo con Kotler y Armstrong (2003), comprende los esfuerzos por cambiar el comportamiento público por otro que la sociedad estime deseable. Giuliani, Montero, Zambon \& Lima (2012) coinciden en ese enfoque y lo amplían al campo social y de responsabilidad. Mientras que Segui (2012) lo aplica al campo familiar. Por otra parte, Pérez romero (2006) amplía la temática al incorporar temas como la población adaptante en lo concerniente a proyectos sociales: cómo convencer a la población de que acepte estos proyectos. Para Fragoso (2014) el marketing puede ser utilizado para alcanzar el bienestar social. , para lo cual el sector público, así como el social y el no lucrativo, pueden contribuir a ese propósito.

Los especialistas en el marketing social provocan cambios positivos mediante incentivos, facilitación o promociones.

Contando con lo que el marketing social significa y cuáles son sus líneas de actuación, es importante a la hora de plantear estrategias para este tipo de marketing la ayuda de consultorías para potenciar los puntos fuertes de la estrategia buscando de esta manera una diferenciación frente a las otras marcas, de una manera ordenada y coherente. Con el fin de cambiar el cambiar el comportamiento que se tiene por otro más aceptable para la sociedad. 
Liderazgo: Según establece Maxwell (2012), el liderazgo representa la facultad de mejorar a las personas de un área, a través de la guía u orientación de un líder, que define como aquel que tiene esa capacidad de influencia a través de la cual sus subordinados mejoran sus aptitudes y capacidades. Covey (1997) hace un extenso estudio de los hábitos que hacen un líder por medio de la obtención de los hábitos efectivos.

Otros conceptos establecen que los líderes se encuentran en permanente evolución personal, ocupándose a la vez de estimular a los demás hacia su propia evolución, por medio de la habilidad o capacidad que poseen para transmitir su enfoque, inspirando o motivando a todos aquello que están a su cargo (individuo, grupos, áreas, etc.) logrando su aprobación y consecuente adhesión, para alcanzar las metas fijadas por la organización.

Para Blanchard y Oncken (1990) los líderes pueden contar con éxito a corto plazo si se centran exclusivamente en alcanzar unos objetivos marcados de antemano, pese a descuidar, sin embargo, factores de estabilidad y prosperidad organizacional tales como la moral o la buena predisposición de los empleados. En cambio, un liderazgo de nivel superior es aquel que se preocupa tanto del desarrollo del personal como del rendimiento de la organización, y consigue que la satisfacción de la plantilla sea mayor y los resultados más duraderos. Liderar es entendido aquí como un proceso de obtención de resultados en el que se actúa con respeto, atención e imparcialidad hacia todos los implicados en el mismo. En cuando a liderazgo educativo, Sierra (2016) lo considera vital para el emprendimiento sostenible. Mientras que, Largacha-Martín y Sierra-Miguéz (2012) argumentan acerca de la necesidad o no de contar con un líder y que este debe contar con la habilidad, capacidad y sensibilidad para poder erigirse dentro de un grupo humano.

\section{Conclusiones}

De este artículo se puede concluir que el liderazgo es la pieza clave para tener mejores oportunidades en el ámbito laboral y personal (Pautt, 2011). Esta cualidad permitirá tener excelentes habilidades sociales, obtener influencia en las personas, actitud positiva, que hoy en día se requiere mucho de ella, ya que la juventud se está llenando de problemas y quejas que no saben cómo resolver. Un mejor liderazgo permitirá tener mayor seguridad en sí mismos, lo que ayudará a tomar decisiones y delegar funciones para ser más proactivos en un equipo de trabajo.

Es por ello que la universidad debe formar planes estratégicos para promover el liderazgo en la comunidad educativa de pre-grado en base al marketing social y poder genera un mayor impacto en los alumnos. Entre ellos se tiene:

Contar la historia de un alumno que haya conseguido desarrollar su liderazgo, es fundamental para influir en su misma comunidad.

Desarrollar en los docentes hábitos de liderazgo para que sean aplicados a los alumnos en cada materia.

Promover eventos acerca del liderazgo en los alumnos. 


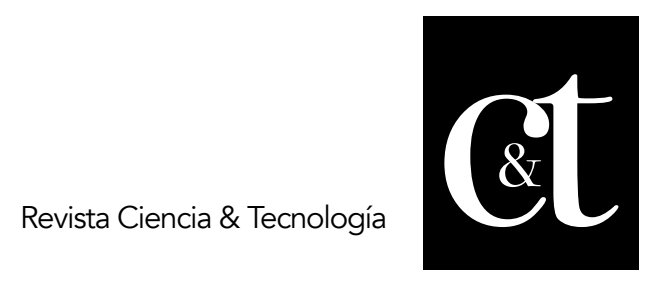

No. 22, 30 de abril de 2019

ISSN impreso: 1390 - 6321

ISSN online: 2661 - 6734

Premiar a los alumnos que realicen actividades para fomentar el liderazgo en sus compañeros. Explicar el impacto positivo que genera el liderazgo en la comunidad educativa de pre-grado

Estos son algunos de los temas que se pueden trabajar para crear conciencia en cada persona que estudia pre-grado, el cambio, autodesarrollo, mejoramiento de las relaciones interpersonales, son cualidades que podrían aumentar si se promueve las estrategias de marketing social correspondientes. Con esto se obtendrá un efecto mayor en la comunidad estudiantil, ganando una fidelización en los clientes que es igual a alumnos.

\section{Referencias bibliográficas}

Blanchard, K. and Oncken, W. (1990). El Ejecutivo al minuto y la organización del tiempo. 385, Barcelona, España: Editorial Grijalbo

Covey, S. (1997). Los 7 hábitos de la genta altamente efectiva. Estados Unidos de América. Editorial Paidós Ibérica.

El Universo, Problemas de las universidades públicas, recuperado de: https://www.eluniverso.com/opinion/2014/08/13/nota/3395376/problemasuniversidades-publicas

Fragoso Bolívar, P. (2014). El Marketing Aplicado al Ámbito Social, Tesis de grado. Universidad Rey Juan Carlos. España. Recuperado de: https://eciencia.urjc.es/bitstream/handle/10115/12221/TFG_FragosoBol\%C3\% ADvarPablo_Fe brero_13-14.pdf?sequence=1\&isAllowed $=y$

García, J. (2010). El Marketing y su origen a la orientación social, Questao. Vol. 16(1), pp.61-77.Recuperado de:https://dialnet.unirioja.es/descarga/articulo/6067049.pdf

Giuliani, C., Monteiro, T., Zambon, M., Betanho, C. \& Lima;Faria, L. (2012), El marketing social, el marketing relacionado con las causas sociales y la responsabilidad social. Invenio. Vol 16(1), pp. 61-77. social, el marketing relacionado con causas sociales y la responsabilidad social empresarial, recuperado de: http://www.redalyc.org/pdf/877/87724146003.pdf

Kotler, P. \& Armstrong, G. (2003). Fundamentos de Marketing. USA: Pearson.

Mccarthy, J. (1960). Basic Marketing. Homewood, USA: Irwin Inc.

Maxwell, G. (2007). Líder de 360. Estados Unidos de América. Editorial Caribe, Inc.

Maxwell, J. (2012). Los 5 niveles de liderazgo: demostrados pasos para maximizar su potencial. New York, USA: Center Street

Pautt, G. (2011). Liderazgo y dirección. Revista Facultad de Ciencias Económicas. V. XIX (1), pp. 213-228. Recuperado de: http://www.redalyc.org/pdf/909/90922732013.pdf

Pérez Romero, L. (2006). Marketing Social. México, México: Editorial PRENTICE HALL MEXICO

Segui, G. (2012). El marketing social como alternativa para combatir La violencia intrafamiliar. Recuperado de:

https://dugidoc.udg.edu/bitstream/handle/10256/8754/Article1- 


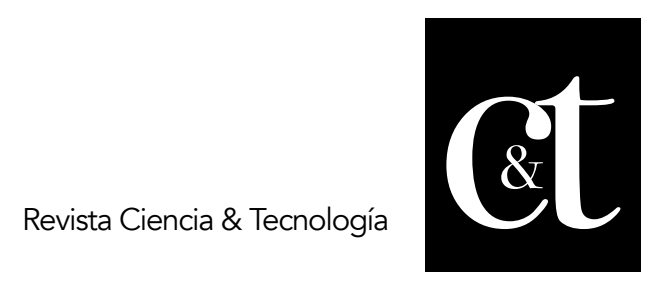

No. 22, 30 de abril de 2019

ISSN impreso: 1390 - 6321

ISSN online: 2661 - 6734

\section{p11.pdf?sequence $=1$}

Sierra, G. (2016), Liderazgo educativo en el siglo XXI, desde la perspectiva del emprendimiento sostenible, recuperado de:

http://www.scielo.org.co/pdf/ean/n81/n81a06.pdf

Largacha-Martín, C. y Sierra-Miguéz, M. (2012). Se necesita el líder? Real Revista de estudios avanzados del liderazgo, Vol. 1(1), pp. 14-25. Recuperado de: https://www.regent.edu/acad/global/publications/real/vol1/num1/REAL_Vol1Nu m1.pdf 